\title{
Records of Galerucinae (Coleoptera, Chrysomelidae) from the Indian subcontinent. Part 1.
}

\author{
Hans Silfverberg
}

Silfverberg, H. 1990: Records of Galerucinae (Coleoptera, Chrysomelidae) from the Indian subcontinent. Part 1. - Entomol. Fennica 1:201-207.

Records are given for 29 species of Oidini, Galerucini, Metacyclini and Hylaspini from Pakistan, northern India, Nepal and Bhutan. Meristata quadrifasciata interrupta (Kollar \& Redtenbacher) is considered subspecifically distinct, Galerucella placida Baly is considered separate from G. grisescens (Joann.), and the status of Nepalogaleruca elegans angustilineata Kimoto \& Takizawa is discussed.

Hans Silfverberg, Zoological Museum, N. Järnvägsg. 13, SF-00100 Helsingfors, Finland

In recent years the insect fauna of the Himalayan area has been quite intensively studied by many scientific institutions. Material collected by such expeditions has resulted in many reports concerning various insect groups. The material upon which this report is based comes from two institutions, the Biosystematics Research Institute in Ottawa, Canada, and the Natural History Museum in Basel (abbreviated as MB). Most of the Ottawa material was collected by the Canadian Nepal Expedition in 1967, in the lists abbreviated as CNE. Additionally a few specimens from the Natural History Museum, London $(\mathrm{BMNH})$, are included. The data for the species are otherwise in the form given on the labels.

\section{Records}

OIDINI

Oides flava (Olivier, 1807)

Nepal: Nepal, nr. Simra, Adhabhar, 600 ft., 27.8.1967, CNE (8 exx.).
Previous records — India: Maharashtra, Tamil Nadu, Arunachal Pradesh. Bangladesh. Kampuchea. Laos. Vietnam. China (Hainan). Malaysia. Indonesia. Philippines. Not previously reported from Nepal.

Oides livida (Fabricius, 1801)

India: Sikkim 77, Bhakta B., Sara Khola, 870 m (Rangeli River), 18.4., 1 ex. (MB).

Previous records - India: W.Bengal (Darjeeling Distr.), Sikkim, Meghalaya, Manipur. Nepal. Bhutan. Bangladesh. Burma. Thailand. Laos. Vietnam. China. Malaysia. Indonesia.

\section{Oides scutellata (Hope, 1831)}

Pakistan: Swat, Pak., 1978, W.Wittmer, Miandam, 3.VI., 1800/2300 m (2 exx.) (MB). — India: India, U.P., 1978, W.Wittmer, Bhimtal, 1-15.5., 1400/1500 m (2 exx.) (MB); India, U.P., 1978, W.Wittmer, Bhowali, 12.5., 1500/ 1600 m (1 ex.) (MB); India, U.P., 1978, W.Wittmer, Nalnital [The name is given as Nalnital, which presumably is a printing error for Nainital], 13.V., 1800/2000 m (4 exx.) (MB); India, U.P., 1978, W.Wittmer, Nalnital, 14.V., 1800/ 2000 m (13 exx.) (MB); India, U.P., 1978, W.Wittmer, Chaurengi 23.5., 2200-2500 m (1 ex.) (MB).

Previous records - Pakistan. India: Himachal Pradesh, Uttar Pradesh (Kumaon), Meghalaya. Nepal. Bangladesh. 


\section{GALERUCINI}

Periclitena vigorsii (Hope, 1831)

Nepal: Nepal, 4400', Kathmandu, 20.6.1967, CNE (3 exx.); id., 4600', 20.6 .1967 (2 exx.); Nepal, Ktmd., Godavari, 6000', 12.7.1967, CNE (1 ex.); id., 5000', 15.7.1967 (1 ex.); Nepal, Bhakta B., Trisuli, 20.VI., 570-1200 m (1 ex.) (MB); O.Nepal, 1980, W.Wittmer, Num - Hedangna, 1500-750-1100 m, 26.5. (2 exx.) (MB); O.Nepal, 1980, W.Wittmer, Lamobagar Gao - Hedangna, 1000-15001100 m, 4.6. (1 ex.) (MB); E.Nepal, Arun V., M.Brancucci, Num, 1550 m, 5-6.VI.1983 (1 ex.) (MB); E.Nepal, Arun V., M.Brancucci, Lamobagar - Gola, 1400 m, 8-14.VI. 1983 (2 exx.) (MB); E.Nepal, Arun V., M.Brancucci, Arun R., 800 m, Hedangan - Num, 16.VI.1983 (1 ex.) (MB). - India: Darjeeling Distr., W.B. India, 1978, Pedong, 15.VIII., 800/1200 m (2 exx.) (MB); India, Bhakta Bahadur, Umg. Kalimpong, 8.5.1981, $600 \mathrm{~m}$ (1 ex.) (MB); Darjeeling Distr., India, Bhakta B., Kalee, 600 m, 9.X.81 (1 ex.) (MB); Darjeeling Distr., India, Bhakta B. (1 ex.) (MB); Sikkim, Bhakta B., Mangon, 2.9.1977 (2 exx.) (MB); Sikkim, Bhakta B., Pelling, 2100 m, 10.IV.1978 (1 ex.) (MB).

Previous records - Pakistan. India: Punjab, Kerala, Bihar, W.Bengal, Sikkim, Meghalaya, Arunachal Pradesh, Manipur. Nepal. Bangladesh. Burma. Thailand. Lacs. Vietnam. China (Hainan). Malaysia.

\section{Doryxena grossa (Hope, 1831)}

Nepal: Nepal, 4400', Kathmandu, 2-7.9.1967, CNE (1 ex.).

Previous records - India: "Assam". Nepal. China.

\section{Sastroides purpurascens (Hope, 1831)}

Nepal: Nepal, 1977, Wittmer, Brancucci, Balaju, 1300-1370 m, 23.5. (1 ex.) (MB); Nepal, Kathmandu V., M.Brancucci, Godawari, 1500 m, 17.V.1983 (1 ex.) (MB); Nepal, Kathmandu V., M.Brancucci, Balaju, 1400 m, 20.V.1983 (1 ex.) (MB); E.Nepal, Arun V., M.Brancucci, Arunthan $1300 \mathrm{~m}$ - Chichila $1950 \mathrm{~m}, 29$. V.1983 (1 ex.) (MB).

Previous records - India: Sikkim. Nepal. Burma. Thailand. Laos.

\section{Menippus cervinus (Hope, 1831)}

India: India, U.P., M.Brancucci, Naugaon, 1100 m, 11.6.1981 (38 exx.) (MB).

Previous records - India: Uttar Pradesh. Nepal. Burma. Thailand. Laos. Vietnam. China.

\section{Diorhabda lusca Maulik, 1936}

India: Indien, Kashmir, Aspöck, Rausch, 8.VII.1980, Ekala - Sonder, 1800-2000 m (1 ex.) (MB); India, U.P.,
1981, M.Brancucci, Bhimtal, 20.V., 1300-1500 m (1 ex.) (MB); India, U.P., 1981, M.Brancucci, Barkot, 5-12.6., 1100-1200 m (4 exx.) (MB); India, U.P., M.Brancucci, Naugaon, 1100 m, 11.6.1981 (38 exx.) (MB).

Previous records - Pakistan. India: Himachal Pradesh, Uttar Pradesh (Dehra Dun).

\section{Atysa marginata (Hope, 1831)}

India: Jammu, 1980, W.Wittmer, Patni Top, $2000 \mathrm{~m}$, 2.7. (32 exx.) (MB); India, U.P., 1981, M.Brancucci, Chaubattia, 25.5., 1900-2100 m (6 exx.) (MB); India, U.P., M.Brancucci, Mussoorie, 2000 m, 30.5.-4.6.1981 (1 ex.) (MB). - Nepal: O.Nepal, 1980, W.Wittmer, Lamobagar Gao, 1400 m, 28-31.5. (1 ex.) (MB).

Previous records - Pakistan: Punjab. India: Uttar Pradesh (Kumaon), Meghalaya, Arunachal Pradesh, "Assam”. Nepal. Burma. Vietnam. China.

\section{Galerucella placida Baly, 1878}

Nepal: Nepal, Ktmd., Godavari, 5000', 16.8.1967, CNE (8 exx.); Nepal, nr. Simra, Adhabhar, $600 \mathrm{ft}$, 23.8.1967, CNE (3 exx.); id., 26.8 .1967 (5 exx.); id., 27.8.1967 (3 exx.); Nepal, nr. Birganj, Lothar, 450 ft., 8.9.1967, CNE (1 ex.); Nepal, $27^{\circ} 58^{\prime}$ N. $88^{\circ} 00^{\prime}$ E, 11000 ft., 5.5.1967, CNE (1 ex.).

Previous records - Afghanistan. Pakistan: Punjab. India: Kashmir, Uttar Pradesh, Tamil Nadu, Bihar, W.Bengal. Nepal. Bangladesh. Burma. Thailand. Laos. Vietnam. Indonesia.

Kimoto (1989) synonymized this species with G. grisescens (Joann.). At least the European $G$. grisescens specimens differ from $G$. placida (cf. Silfverberg 1974). The East Asian Galerucella distincta (Baly) has been considered a subspecies or a synonym of $G$. grisescens (Gressitt \& Kimoto 1963), but I have not seen any data from a comparison between it and the Indian species.

\section{Xanthogaleruca luteola (Müller, 1766)}

Galerucella luteola var. bicarinata Ogloblin, 1936:108. Pyrrhalta luteola bicarinata, Lopatin 1984:336.

India: Jammu, 1980, W.Wittmer, Sirshi - Hanzal, 1700-2100 m, 8.7. (1 ex.) (MB); Jammu, 1980, W.Wittmer, Yourdu, 16.7., 2150-2400 m (1 ex.) (MB).

Previous records - India: Kashmir. Europe. North Africa. Asia Minor. Iran. Afghanistan. Siberia. North America.

Only a few specimens have been reported from Kashmir, and the question whether they should be given status as a separate subspecies, $X$. l. bicarinata, must be deferred until more material has been studied. 


\section{Pallasiola theoneiformis Mandl, 1970}

India: Kashmir, 1976, W.Wittmer, Gulmarg, 1-3.7., 2650-3000 m (25 exx.) (MB); Kashmir, 1976, W.Wittmer, Yusmarg, 5.7., 2300-2400 m (20 exx.) (MB); Jammu, 1980, W.Wittmer, Sarkandu - Inchan, 2350-2500 m (2 exx.) (MB); Indien, Kashmir, Aspöck, Rausch, 1980, Yourdu - Sarkandu, 17.7., 2350 m (1 ex.) (MB); Indien, Kashmir, Aspöck, Rausch, 1980, Inchan, 19.7., 2500-2800 m (1 ex.) (MB).

Only known from Kashmir.

\section{Pseudadimonia variolosa (Hope, 1831)}

Nepal: Nepal, Ktmd., Godavari 5000', 12.8.1967, CNE (2 exx.); Nepal, Ktmd., Balaju, 4500 ft., 10.6.1967, CNE (1 ex.).

Previous records - India: W.Bengal (Darjeeling Distr.), Sikkim, Meghalaya, Assam, Arunachal Pradesh, Nagaland, Manipur. Nepal. Bhutan. Bangladesh. Burma. Thailand. Laos. Vietnam. China.

\section{MetaCyCLini}

\section{Pseudoides flavovittis (Motschulsky, 1858)}

Nepal: Nepal, Lothar nr. Birganj, 450', 29.8.1967, CNE (8 exx.); id., 31.8 .1967 (13 exx.); id., 1.9.1967 (19 exx.); id., 10.9.1967 (2 exx.); id., 14.9 .1967 (1 ex.); id., 17.9.1967 (12 exx.); id., 19.9.1967 (1 ex.); id., 29.8.5.9.1967, Malaise Trap No 25 (1 ex.). - Bhutan: Bhutan, 1981, Bhakta B., Phuntsholing, 2/400 m, 3.IX. (56 exx.) (MB).

Previous records - Nepal. Bhutan. Burma. Thailand. Kampuchea. Laos. Vietnam.

\section{Hylaspini (Sermylini)}

\section{Dercetina histrio (Baly, 1879)}

India: India, Darjeeling D., Bhakta B., Khani Khola, 1.IV.83, $600 \mathrm{~m}$ (4 exx.) (MB). ladesh.

Previous records - India: W.Bengal, "Assam". Bang-

\section{Aplosonyx chalybeus (Hope, 1831)}

Nepal: Nepal, Ktmd., Godavari, 6000', 6.7.1967, CNE (1 ex.); Danda Pokhar, 1600-2500 m, 1.6., Nepal, 1977, M.Brancucci (1 ex.) (MB); Nepal, 1978, Bhakta B.Ch., Manigow, 10.VI., 1200-1900 m (1 ex.) (MB); Nepal, Bhakta B., Junbesi, 2700 m, 25.5.79, Khumbu (1 ex.) (MB); Nepal, 1979, Bhakta B.Ch., Chandan Bari, 3350 4250 m, VI. (1 ex.) (MB); O.Nepal, 1980, W.Wittmer, Num Hedangna, 1500-750-1100 m, 26.5. (1 ex.) (MB);
O.Nepal, 1980, W.Wittmer, Hedangna - Shedawa, 5.6., 1000-800-1700 m (1 ex.) (MB); O.Nepal, 1980, W.Wittmer, Phulchoki, 23.6., 1500-1700 m (2 exx.) (MB); Nepal, Kathmandu V., M.Brancucci, Godavari 1500 m, 17.V.1983 (1 ex.) (MB); id., 22-25.VI.1983 (2 exx.) (MB); E.Nepal, Thamur V., M.Brancucci, Dhankuta, 23.V.1983 (1 ex.) (MB); E.Nepal, Arun V., M.Brancucci, Mure, 2000 m, 2-8.VI.1983 (1 ex.) (MB); E.Nepal, Arun V., M.Brancucci, Num 1550 m, 5-6.VI.1983 (4 exx.) (MB); E.Nepal, Arun V., M.Brancucci, Lamobagar, 1400 m, 8.VI.1983 (3 exx.) (MB); E.Nepal, Arun V., M.Brancucci, Lamobagar - Gola, 1400 m, 8-14.VI.1983 (7 exx.) (MB); E.Nepal, Arun V., M.Brancucci, Lamobagar $1400 \mathrm{~m}$ Hedangna (Arun), 15.VI.83, 1400-800 m (1 ex.) (MB). - India: Sikkim, Bhakta B., Sisne, S.Gangtok, 930 m, 14.4.1977 (1 ex.) (MB); Sikkim, 1977, Bhakta B., Rangeli River, 950 m, 17.4. (4 exx.) (MB); Sikkim, 77, Bhakta B., Sara Khola 870 m (Rangeli River), 18.4. (1 ex.) (MB); Sikkim, 77, Bhakta B., Rani Puli, 22.4., S.Gangtok (3 exx.) (MB); Sikkim, 77, Bhakta B., Chhuba Khola nr. Sintam, 25.4. (2 exx.) (MB); Sikkim, 77, Bhakta B., Rezi Bazar nr. Sintam, 26.4. (1 ex.) (MB); Sikkim, Bhakta B., Mangon, 2.9.1977 (2 exx.) (MB); Sikkim, Bhakta B., Tuing, 4.9 .77 (2 exx.) (MB); Sikkim, Bhakta B., Fadung, 6.9.1977 (1 ex.) (MB); Fadung - Boxapull, Sikkim, 7.9.77, Bhakta B. (15 exx.) (MB); Sikkim, 77, Bhakta B., Diukchu, 10.9. (15 exx.) (MB); Sikkim, Bhakta B., Rangpo 680 m, 3.VIII.1981 (1 ex.) (MB). Kalimpong Umg., Bhakta Bahadur, 77, Khani Khola, 490 m, W.Kalimpong, 2.5. (1 ex.) (MB); Umg. Kalimpong, Darjeeling Distr., 10.5.77, 1530 m (1 ex.) (MB); Distr. Darjeeling, India, W.Wittmer, Singla 450 m, 19.IV.1978 (2 exx.) (MB); Darjeeling Distr., W.B., India, Pankha Sari, 1300 m, 8.VIII.78 (17 exx.) (MB); Darjeeling Distr., India, Bhakta B., Goroo Bethan, 840 m, 21.IV.1979 (1 ex.) (MB); Darjeeling Distr., India, Bhakta B., Bom Busty, 1050 m, 6.5.1981 (3 exx.) (MB); Kalimpong, Darjeeling Distr., India, 5.VII.1981, Bhakta Bahadur (2 exx.) (MB); Darjeeling Distr., India, Bhakta B., Tista $230 \mathrm{~m}, 16.7 .1981$ (6 exx.) (MB); Darjeeling Distr., India, Bhakta B., 81 (2 exx.) (MB); Darjeeling D., India, Bhakta B., Monsong, 1100-1300 m, 6-7.V.1983 (1 ex.) (MB); Darjeeling Distr., India, Bhakta B. (4 exx.) (MB). - Bhutan: Bhutan, Dorjee Khandu, Chasilakha, $6425^{\prime}, 1978$ (3 exx.) (MB); Bhutan, Dorjee Khandu Dukpa, 1280 m, 7.IV.1979 (6 exx.) (MB).

Previous records - India: W.Bengal (Darjeeling Distr.), Sikkim, Meghalaya, "Assam". Nepal. Bhutan. Bangladesh. Burma. Vietnam. China (Tibet).

\section{Sphenoraia bicolor (Hope, 1831)}

Pakistan: Pakistan, 1977, Wittmer, Brancucci, Lake Saiful, 21.VI., Muluk, 3050 m (1 ex.) (MB). - India: India, U.P., 1981, M.Brancucci, Bhimtal, 20.V. 1300-1500 m (1 ex.) (MB); India, U.P., 1981, M.Brancucci, Chaubattia, 25.5., 1900-2100 m (1 ex.) (MB); India, U.P., M.Brancucci, Kempty, 1200 m, 3.6.1981 (1 ex.) (MB); India, U.P., 1981, M.Brancucci, Barkot, 5-12.6., 
1100-1200 m (2 exx.) (MB); India, U.P., M.Brancucci, Naugaon, 1100 m, 11.6.1981 (3 exx.) (MB); India, U.P., M.Brancucci, Gangani, 1250 m, 13-20.6.1981 (3 exx.) (MB). - Nepal: Nepal, Balaju nr. Ktmd., 4500 ft., 26.4.1967, CNE (2 exx.); Nepal, Kathmandu, 2.5.1967, CNE (1 ex.); Nepal, Ktmd., Patibhanjyang, 6-7500', Pastures, 12.6.1967, CNE (1 ex.); Nepal, Ktmd., Godavari, 5000', 20.7.1967, CNE (1 ex.); id., 29.7.1967 (4 exx.); id., 4-7.8.1967 (4 exx.); id., 6.8 .1967 (1 ex.); id., 12.8.1967 (1 ex.); id., 16.8.1967 (1 ex.); Nepal, nr. Birganj, Lothar, 450 ft., 14.9.1967, CNE (1 ex.); Nepal, 1978, Bhakta B., Manigow, 10.VI., 1200-1900 m (1 ex.) (MB); Nepal, 1978, Bhakta B.Ch., Trisuli, 20.VI., 570-1200 m (1 ex.) (MB); Nepal, 1978, Bhakta B., Chadam Bari, 3350 m, 22.VI. (1 ex.) (MB); O.Nepal, 1979, Bhakta B., Sete, 28.V., 2350 m (1 ex.) (MB); O.Nepal, 1980, W.Wittmer, Lamobagar Gao, 1400 m, 28-31.5. (1 ex.) (MB); O.Nepal, 1980, W.Wittmer, Navagaon - Num, 1900-700-1500 m, 16.6. (1 ex.) (MB); Nepal (Prov. Bagmati), Barabhise, $1600 \mathrm{~m}$, 1.5.1981, Löbl \& Smetana (1 ex.) (Mus. Ottawa); Nepal, Kathmandu V., M.Brancucci, Balaju, 1400 m, 20.V.1983 (1 ex.) (MB). - India: Sikkim, Bhakta B., Rangeli River, 900 m, 15.4.1977 (2 exx.) (MB); id., 19.4.1977 (3 exx.) (MB); Sikkim, Bhakta B., Reay Khola S.Gangtok, 21.4.1977 (1 ex.) (MB); Sikkim, Bhakta B., Chhuba Khola nr. Sintam, 25.4.1977, 670 m (4 exx.) (MB); Sikkim, 77, Bhakta B., Boxapull - Diukchu, 9.9. (1 ex.) (MB); Sikkim, 77, Bhakta B., Diukchu, 10.9. (6 exx.) (MB); Sikkim, Bhakta B., Choka - Yoksam, 2100 m, 6.IV.78 (1 ex.) (MB); Sikkim, Bhakta B., Gyalzing, 1600 m, 12.IV.78 (2 exx.) (MB); Sikkim, Bhakta B., Reshi, 400 m, 15.IV.1978 (1 ex.) (MB); Sikkim, Bhakta B., Reshi - Yortang, 400 m, 17.IV.1978 (2 exx.) (MB); Sikkim, 1981, Bhakta B., Malli, 460 m, 20.VII. (1 ex.) (MB). Darjeeling Distr., Umg. Kalimpong, Bhakta Bahadur, 3.IV.1977 (1 ex.) (MB); Sherpa Gau nr. Kalimpong, 1380 m, Bhakta Bahadur, 9.5.1977 (1 ex.) (MB); Darjeeling Distr., India, Bhakta B., Khani Khola, 490 m, W Kalimpong, 26.IV.79 (1 ex.) (MB); Darjeeling D., India, Bhakta B., Tashiding N Kalimpong, 1000 m, 27.V.1979 (2 exx.) (MB); Darjeeling D., India, Bhakta B., Shorang, 1300 m, 4.V.1979 (1 ex.) (MB).

Previous records - Pakistan. India: Himachal Pradesh, Uttar Pradesh, Maharashtra, Kerala, W.Bengal, Sikkim, Meghalaya. Nepal. Bangladesh. Burma.

\section{Sphenoraia rutilans (Hope, 1831)}

India: India, U.P., 1978, W.Wittmer, Bhimtal, 1400 m, VI-VII (1 ex.) (MB); India, Himalaya, U.P., District Dehra Dun, 2000 m, Mussoorie, 1-4.6.1981, leg. C.Holzschuh (2 exx.) (MB); India, U.P., 1981, M.Brancucci, Barkot, 5-12.6., 1100-1800 m (14 exx.) (MB); India, U.P., M.Brancucci, Gangani, 1250 m, 13-20.6.81 (1 ex.) (MB); India, U.P., M.Brancucci, Kharidy, 1300 m, 17.6.1981 (1 ex.) (MB). - Nepal: Nepal, Kathmandu V., M.Brancucci, Balaju, 1400 m, 20.V.1983 (1 ex.) (MB); E.Nepal, Arun V., M.Brancucci, Phalicot, 13.VI.83, 550 m (1 ex.) (MB);
O.Nepal, 1980, W.Wittmer, Arunthan - Chichila, 1300-1950 m, 23.5. (1 ex.)) (MB). - India: Sikkim, Bhakta B., Rangeli River, 900 m, 19.IV.77 (2 exx.) (MB); Sikkim, 77, Bhakta B., Anu Khola nr. Rani Puli, 23.4. (1 ex.) (MB); Sikkim, 1981, Bhakta b., Melli, 460 m, 20.VII. (1 ex.) (MB). Distr. Darjeeling, India, W.Wittmer, Lopchu, 9.5.1975 (3 exx.) (MB); Darjeeling Distr., W.B., India, 1976, Lopchu, 3.5.76 (3 exx.) (MB); Kalimpong Umg., Bhakta Bahadur, 6.4 .77 (1 ex.) (MB); Darjeeling Distr., Bhakta B.Ch., Shorang, 1300 m, 26.VII.1978 (1 ex.) (MB); Darjeeling Distr., India, Bhakta B., Khani Khola, 490 m, W.Kalimpong, 26.IV.79 (1 ex.) (MB); Darjeeling Distr., India, Bhakta B., Balie, 780 m, 2.5.1981 (2 exx.) (MB); Darjeeling Distr., Kalimpong, 5.VII.81, Bhakta Bahadur (2 exx.) (MB); India, Bhakta Bahadur, Tar Khola nr. Maelli, 540 m, 4.5 .77 (1 ex.) (MB).

Previous records - Pakistan. India: Kashmir, Himachal Pradesh, Uttar Pradesh, W.Bengal, Sikkim, Manipur, Meghalaya, Arunachal Pradesh. Nepal. Bhutan. Bangladesh. China.

\section{Gallerucida indica Harold, 1880}

Nepal: Nepal, Ktmd., Godavari, 6000', 2.8.1967, CNE (2 exx.).

Previous records - India: W.Bengal (Darjeeling Distr.). Nepal. Bangladesh.

\section{Hylaspes dohrni Duvivier, 1885}

Nepal: O.Nepal, 1980, W.Wittmer, Num - Hedangna, 1500-750-1100 m, 26.5. (1 ex.) (MB); E.Nepal, Arun V., M.Brancucci, Arunthan $1300 \mathrm{~m}$ - Chichila $1950 \mathrm{~m}$, 29.V.1983 (1 ex.) (MB).

Previously only recorded from Burma.

\section{Hylaspes longicornis Baly, 1865}

Nepal: Nepal, Kathmandu V., M.Brancucci, Godawari, 1500 m, 17.V.1983 (2 exx.) (MB); id., 22-25.VI.1983 (2 exx.) (MB); Nepal, Kathmandu V., M.Brancucci, Burhanilkanth, 1440-750-1100 m, 16.VI.1983 (1 ex.) (MB); O.Nepal, Bhakta B., Jubing, 1300 m, 20.VI.1979 (2 exx.) (MB); O.Nepal, 1980, W.Wittmer, Chichila - Mure, 1900 m, 24.5. (1 ex.) (MB); O.Nepal, 1980, W,Wittmer, Mure - Num, 25.5., 1900-1500 m (1 ex.) (MB); O.Nepal, 1980, W.Wittmer, Num - Hedangna, 1500-750-1100 m, 26.5. (1 ex.) (MB); O.Nepal, 1980, W.Wittmer, Hatiya Lamobagar Gao, 2.6., 1550-1000 m (1 ex.) (MB); O.Nepal, 1980, W.Wittmer, Navagaon - Num, 1900 700-1500 m, 16.6. (1 ex.) (MB); O.Nepal, 1980, W.Wittmer, Phulchoki, 25.6., 1500-1600 m (1 ex.) (MB); E.Nepal, Arun V., M.Brancucci, Chichila 1950 - Mure 2000 m, 1.VI.1983 (2 exx.) (MB); E.Nepal, Arun V., M.Brancucci, Num, 1550 m, 5-6.VI.1983 (2 exx.) (MB); E.Nepal, Arun V., M.Brancucci, Mure 2000 - Arunthan 1300 m, 9.VI.1983 (1 ex.) (MB); E.Nepal, Arun V., 
M.Brancucci, Lamobagar Gola, 1400 m, 14.VI.1983 (2 exx.) (MB). - India: Sikkim, Bhakta B., Rangeli River, 900 m, 15.4.77 (2 exx.) (MB). Darjeeling Distr., India, Bhakta B., Goroo Bathan, 840 m, 21.IV.1979 (1 ex.) (MB); Darjeeling Distr., India, Bhakta B., Tista, $230 \mathrm{~m}, 10.5 .1981$ (1 ex.) (MB).

Previous records - India: Sikkim, "Assam". Nepal. Bhutan. Bangladesh. Burma. Thailand. Laos.

\section{Meristata dohrni (Baly, 1861)}

Nepal: Nepal, Ktmd., Godavari, 6000', 12.7.1967, CNE (1 ex.); id., 14.7.1967 (2 exx.); id., 17.7.1967 (1 ex.); id., 18.7.1967 (1 ex.); id., 3.8.1967 (1 ex.); Nepal, Kathmandu V., M.Brancucci, Godavari, 1500 m, 17.V.1983 (10 exx.) (MB); Nepal, 1978, Bhakta B.Ch., Manigow, 10.VI., 1200-190ก 'm (3 exx.) (MB); O.Nepal, 1979, Bhakla , 14.VI., $2900 \mathrm{~m}$ (2 exx.) (MB); O.Nepal, lyou, vv.vvittmer, Chichila - Mure, 1900 m, 24.5. (1 ex.) (MB); O.Nepal, 1980, W.Wittmer, Hong Gaon - Kemathanka, 2900 m, 30.5. (1 ex.) (MB); O.Nepal, 1980, W.Wittmer, V., Arun V., 1/2000 m (1 ex.) (MB); E.Nepal, Arun V., M.Brancucci, Chichila 1950 - Mure 2000 m, 1.VI.1983 (1 ex.) (MB); E.Nepal, Arun V., M.Brancucci, Mure, 2000 m, 2-8.VI.1983 (2 exx.) (MB); E.Nepal, Arun V., M.Brancucci, Mure, $2000 \mathrm{~m}$ - Num, 1550 m, 4-7.VI.1983 (1 ex.) (MB). - India: Sikkim, Bhakta B., Rishi Khola, 700 m, 3.XII.81 (5 exx.) (MB). - Bhutan: Bhutan, Dorjee Khandu, Chasilakha, 6425', 1978 (2 exx.) (MB).

Previous records - India: W.Bengal (Darjeeling Distr.), Sikkim, Meghalaya, Manipur. Nepal. Bhutan. Burma. China (Tibet).

\section{Meristata fallax (Harold, 1880)}

Nepal: E.Nepal, Arun V., M.Brancucci, Mure, 2000 m, 2-8.VI.1983 (2 exx.) (MB). - India: Sikkim, 1980, Bhakta B., Thenling - Yoksam, 1200-1700 m, 20.VIII. (1 ex.) (MB). Darjeeling D., Ch.J.Rai, Alghera, 2900 m, 25.IV. 1982 (1 ex.) (MB). - Bhutan: Bhutan, Dorjee Khandu, Chasilakha, 6425', 1978 (3 exx.) (MB); Bhutan, Dorjee Khandu D., Chasilakha, VI.1979 (1 ex.) (MB).

Previous records - India: W.Bengal, Sikkim, Arunachal Pradesh. Nepal. Bhutan. Bangladesh.

\section{Meristata pulunini (Bryant, 1952)}

Nepal: Nepal, Bhakta B.Ch., Langtang, 14.VI.79, 3350-3400 m (2 exx.) (MB); O.Nepal, 1980, W.Wittmer, V., Arun V., 1/2000 m (1 ex.) (MB).

Previous records - Nepal. China (Tibet).

Meristata quadrifasciata quadrifasciata (Hope, 1831)

India: India, U.P., F.Smetacek, Bhimtal, 30.V.1981, $1400 \mathrm{~m}$ (1 ex.) (MB); India, U.P., M.Brancucci, Mus- soorie, 2000 m, 30.5.-4.6.1981 (2 exx.) (MB); India, U.P., 1981, M.Brancucci, Barkot, 5-12.6.1981, 1100-1200 m (1 ex.) (MB); India, U.P., M.Brancucci, Gangani, $1250 \mathrm{~m}$, 13-20.6.1981 (3 exx.) (MB); India, U.P., M.Brancucci, Kharidy, 1300 m, 17.6.1981 (1 ex.) (MB). — Nepal: Nepal, Balaju nr. Kathmandu, 4500', 26.4.1967, CNE (5 exx.); Nepal, 4400', Kathmandu, 28.4.1967, CNE (4 exx.); Nepal, Ktmd, Sunderijal, 6-7500', Pastures, 8.5.1967, CNE (1 ex.); Nepal, nr. Ktmd., Gulubhanjyang, 75-8500', Pastures, 9.5.1967, CNE (1 ex.); Nepal, Ktmd., Patibhanjyang, 75-8500', Pastures, 12.6.1967, CNE (1 ex.); Nepal, Ktmd., Godavari, 5000', 27.6.1967, CNE (1 ex.); id., 6.8.1967 (3 exx.); id., 16.8.1967 (1 ex.); Nepal (Prov. Bagmati), below Yardang Ridge NE Barabhise, 3150 m, 4.5.1981, Löbl \& Smetana (2 exx.) (Mus. Ottawa); Nepal, Kathmandu V., M.Brancucci, Godawari, 1500 m, 17.V.1983 (6 exx.) (MB); Nepal, Bhakta B., Junbesi, 2700 m, 25.5.79, Khumbı (2 exx.) (MB); O.Nepal, Bhakta B., Magehan, 1700 m, 25.V.1979 (1 ex.) (MB); O.Nepal, W.Wittmer, Hedangna - Lamobagar Gao, 11/1200 m, 27.5.1980 (1 ex.) (MB); O.Nepal, 1980, W.Wittmer, Nagarkot, 26.6., 18002000 m (2 exx.) (MB); E.Nepal, Arun V., M.Brancucci, Hedangna $1100 \mathrm{~m}$ - Lamobagar 1400 m, 8.VI.1983 (1 ex.) (MB); E.Nepal, Arun V., M.Brancucci, Lamobagar - Gola, 1400 m, 8-14.VI.1983 (6 exx.) (MB); E.Nepal, Arun V. M.Brancucci, Lamobagar - Hedangna (Arun), 15.VI.83, 1400-800 m (1 ex.) (MB). — India: Sikkim, 77, Bhakta B., Sara Khola, 870 m (Rangeli River), 18.4. (1 ex.) (MB); Sikkim, 77, Bhakta B., Rani Puli S Gangtok, 22.4. (1 ex.) (MB).

Previous records - India: Uttar Pradesh, Sikkim. Nepal. Bhutan. Bangladesh. Whether the records from Himachal Pradesh and Punjab (India) belong to this or the following subspecies remains to be checked.

\section{Meristata quadrifasciata interrupta (Kollar \&} Redtenbacher, 1848)

Galleruca interrupta Kollar \& Redtenbacher, 1848:553.

Pakistan: Pakistan, 1977, Wittmer, Brancucci, Murree, 2 C50 m, 12.6. (1 ex.) (MB); W.Wittmer, Murree, 18.VII., 2050 m (1 ex.) (MB); Swat, Pak., 1978, W.Wittmer, Miandam, 3.VI., 1800-2300 m (8 exx.) (MB).

Previous records - Pakistan: N.W.F.P., Punjab. India: Kashmir.

The western specimens of M.quadrifasciata differ clearly from the eastern ones in generally having the anterior transversal band on the elytra interrupted several times (in the eastern ones it is entire). It thus seems appropriate to recognize a western subspecies, for which Redtenbacher's name is available; it will be credited to Kollar \& Redtenbacher because nowhere in the paper was it stated that Kollar described the Lepidoptera and Redtenbacher the Coleoptera. 
Meristata sexmaculata (Kollar \& Redtenbacher, 1848)

India: India, U.P., M.Brancucci, Mussoorie, $2000 \mathrm{~m}$, 30.5.-4.6.1981 (4 exx.) (MB); India, Himalaya, U.P., District Dehra Dun, Mussoorie, 1-4.6.1981, leg. C.Holzschuh (3 exx.) (MB). - Nepal: Nepal, nr. Ktmd., Gulubhanjyang, 75-8500', Pastures, 2.7.1967, CNE (1 ex.); Nepal, Ktmd., Godavari, 5000', 28.7.1967, CNE (1 ex.); id., 6.8.1967 (1 ex.); O.Nepal, Bhakta B., Ilam, 7.X.1978 (1 ex.) (MB); O.Nepal, 1979, Bhakta B., Lamjura, 26.VI., 3200 m, Khumbu (1 ex.) (MB); O.Nepal, 1980, W.Wittmer, Hong Gaon - Kemathanka, 2900 m, 30.5. (1 ex.) (MB); E.Nepal, Arun V., M.Brancucci, Mure, 2000 m, 2-8.VI.1983 (1 ex.) (MB). - India: Sikkim, 77, Bhakta B., Sara Khola, $870 \mathrm{~m}$ (Rangeli River), 18.4. (1 ex.) (MB); Sikkim, 77, Bhakta B., Diukchu, 10.9. (1 ex.) (MB); Sikkim, 1981, Bhakta B., Jortang, 410 m, 4.VIII. (1 ex.) (MB). Umg. Kalimpong, Darjeeling Distr., Pankha Banglo, 9.VIII.1978 (1 ex.) (MB). - Bhutan: Bhutan, Dorjee Khandu, Chasilakha, $6425^{\prime}, 1978$ (1 ex.) (MB).

Previous records - Pakistan. India: Kashmir, Punjab, Uttar Pradesh, Orissa, W.Bengal, Sikkim. Nepal. Bhutan. Bangladesh. China (Tibet).

\section{Meristata spilota (Hope)}

Galleruca spilota Hope, 1831.

Galleruca trifasciata Hope, 1831. Preoccupied by Galleruca trifasciata Fabricius, 1801.

India: India, U.P., M.Brancucci, Mussoorie, $2000 \mathrm{~m}$, 30.5.-4.6.1983 (3 exx.) (MB). — Nepal: Nepal, nr. Ktmd., Gulubhanjyang, 75-8500', Pastures, 2.7.1967, CNE (1 ex.); Nepal, 6-8000', Sunderijal - Patibhanjyang, 3.7.1967, CNE (1 ex.); Nepal (Prov. Bagmati), Gul Bhanjyang, 2600 m, 6.4.1981, Löbl \& Smetana (1 ex.) (Mus. Ottawa); Nepal, Khandbari Distr., above Sheduwa, $300 \mathrm{~m}$, 3.3.1.4.1982, A. \& Z.Smetana (2 exx.) (Mus. Ottawa); Nepal, Khandbari Distr., above Tashigaon, 3100 m, 7-8.4.1982, A. \& Z. Smetana (1 ex.) (Mus. Ottawa); Nepal, 1978, Bhakta B.Ch., Manigow, 10.VI., 1200-1900 m (7 exx.) (MB); O.Nepal, 1978, Bhakta B., Chitra, 19.IX, 1300-2300 m (1 ex.) (MB); O.Nepal, Bhakta B., Chauki, 22.IX.1978 (1 ex.) (MB); O.Nepal, Bhakta B., Mageham, $1700 \mathrm{~m}$, 25.V.1979 (1 ex.) (MB); O.Nepal, 1979, Bhakta B.Ch., Lukla, 14.VI., 2900 m (1 ex.) (MB); O.Nepal, Bhakta B., Chandam Bari, 3350 m, 26.VI.1979 (1 ex.) (MB).

Previous records - Pakistan: N.W.F.P. India: Punjab, Uttar Pradesh, W.Bengal, Sikkim, Assam. Nepal. Bhutan. Bangladesh. China (Tibet).

\section{Leptarthra fasciata Jacoby, 1894}

Nepal: Nepal, Ktmd., Godavari, 5000', 29.7.1967, CNE (14 exx.); id., 6.8.1967 (6 exx.); id., 12.8 .1967 (3 exx.); id., 16.8.1967 (4 exx.). desh.
Nepalogaleruca elegans elegans Kimoto, 1970

Nepal: Nepal, 8700', Bhurumche, 23.6.1967, CNE (2 exx.); Nepal (Prov. Bagmati), Pokhare NE Barabhise, 2800 m, 2.5.1981, Löbl \& Smetana (4 exx.) (Mus. Ottawa); id., 2700 m (1 ex.) (Mus. Ottawa); Nepal, Kathmandu Distr., Phulcoki, 2600 m, 20.4.1982, A. \& Z.Smetana (4 exx.) (Mus. Ottawa); id., 21.4.1982 (6 exx.); nr. Those, 9-11. IV.52, British Nepal Expedition 1952, T.D.Bourdillon, B.M. 1955-802 (1 ex.) (BMNH); O.Nepal, 1979, Bhakta B.Ch., Lukla, 14.VI., 2900 m (1 ex.) (MB); O.Nepal, 1979 , Bhakta B.Ch., Lamjura, 3200 m, Khumbu, 28.VI. (1 ex.) (MB); Nepal, Bhakta B., Ramche, 1800-3350 m, 18.VI. (1 ex.) (MB).

Nepalogaleruca elegans angustilineata Kimoto \& Takizawa, 1972

Nepal: Nepal (Prov. Bagmati), below Thare Pati, 3300 m, 12.4.1981, Löbl \& Smetana (2 exx.) (Mus. Ottawa); Nepal (Prov. Bagmati), Yangri Ridge, Yangri, $4150 \mathrm{~m}$, 24.4.1981, Löbl \& Smetana (1 ex.) (Mus. Ottawa). China: Tibet: Tropde, Rongshar Valley, 12000 ft., 21.VI.1924, Maj. R.W.G.Hingston, Everest Exp. Brit.Mus. 1924-386 (1 ex.) (BMNH); same data but 30.VI.1924 (1 еx.) (BMNH).

Nepalogaleruca elegans is known from Nepal, and from Tibet just across the border. It has mainly been found fairly high in the mountains, although Kimoto (1982) has reported one specimen from a maximum of $1200 \mathrm{~m}$. The species was described from the Solukhumbu district, and apart from this the nominate subspecies has been recorded from the Ramecchap, Lalitpur, Kathmandu and Sindhupalchok districts (Kimoto 1970, Kimoto \& Takizawa 1972, Kimoto \& Takizawa 1981, Kimoto 1982, Kimoto \& Takizawa 1983, Takizawa 1988a and Takizawa 1988b); now there is an addition single record from the Udaipur District. Chen \& Jiang (1981), who reported this species from Zham (= Dram, = Zhangmu) in the Nyalam County of Tibet, also seem to have seen only the nominate subspecies.

The darker subspecies, $N$. e. angustilineata, was described from the Mustang and Parbat districts, and was later also reported from the Nuwakot and Sindhupalchok districts (Kimoto \& Takizawa 1972, Kimoto \& Takizawa 1983, Takizawa 1988b). In Mustang and Parbat the average elevation of the records was $2610 \mathrm{~m}$, in Sindhupalchok it was $3410 \mathrm{~m}$. The two Tibetan specimens in BMNH, which were collected just 
across the border from Solokhumbu, were taken at approximately $3650 \mathrm{~m}$. Again, for the nominate subspecies, the average elevation in Solukhumbu was $3210 \mathrm{~m}$ while in Sindhupalchok it was $2800 \mathrm{~m}$. It therefore seems that when both subspecies are found in an area, they occur at different elevations.

Acknowledgments. I thank Dr. L. LeSage for sending me the material from Ottawa, and Dr. M. Brancucci for providing me with the material from Basel. For assistance at the Natural History Museum in London I wish to thank Mrs. S. L. Shute.

\section{References}

Chen S. C. \& Jiang S. Q. 1981: Coleoptera: Chrysomelidae-Galerucinae. - Insects of Xizang 1:457-489.

Gressitt, J. L. \& Kimoto, S. 1963: The Chrysomelidae (Coleopt.) of China and Korea. Part 2. - Pacific Insects Monogr. 1:301-1026.

Kimoto, S. 1970: A list of the Nepalese Chrysomelid specimens preserved in Zoologische Sammlung des Bayerischen Staates, München. - Khumbu Himal 3: 412-421.

- 1982: The Galerucinae of Nepal, Bhutan and the Northern Territories of India, in the Natural History Museum in Basel, II (Coleoptera: Chrysomelidae). - Entomol. Rev. Japan 37:7-24.

- 1989: Chrysomelidae (Coleoptera) of Thailand, Cambodia, Laos and Vietnam. IV. Galerucinae. - Esakia 27:1-241.
Kimoto, S. \& Takizawa, H. 1972: Chrysomelid-beetles of Nepal, collected by the Hokkaido University Scientific Expedition to Nepal Himalaya, 1968. Part I. - Kontyu 40:215-223.

- 1981: Chrysomelid-beetles of Nepal, collected by the Hokkaido University Scientific Expeditions to Nepal Himalaya, 1968 and 1975. Part III (Coleoptera). Entomol. Rev. Japan 35:51-65.

- 1983: Chrysomelid Beetles of Nepal, Collected by the Himalaya Expedition 1979 of the National Science Museum, Tokyo (Part 1). - Bull. Natn. Sci. Mus., Tokyo, Ser. A 9:83-96.

Kollar, V. \& Redtenbacher, L. 1848: Aufzählung und Beschreibung der von Freiherrn Carl v. Hügel auf seiner Reise durch Kaschmir und das Himaleyagebirge gesammelten Insecten. - In: Hügel, C. V., Kaschmir und das Reich der Siek 4(2):393-564.

Lopatin, I. 1984: Chrysomelidae aus dem Himalaja (Coleoptera). - Entomol. Basiliensia 9:328-339.

Ogloblin, D. A. 1936: Nasekomye Žestkokrylye. XXVI. 1. Listoedy, Galerucinae. - Fauna SSSR 8:1-455.

Silfverberg, H. 1974: The West Palaearctic species of Galerucella Crotch and related genera (Coleoptera, Chrysomelidae). — Notulae Entomol. 54:1-11.

Takizawa, H. 1988a: Chrysomelid Beetles of Nepal, Collected by the Hokkaido University Scientific Expeditions to Nepal Himalaya. Part IV (Coleoptera: Chrysomelidae). - Entomol. Rev. Japan 43:1-16.

- 1988b: Notes on Chrysomelid Beetles (Coleoptera, Chrysomelidae) of India and its Neighboring Areas. Part 7. - Kontyu 56:534-552.

Received 11.XII.1989 